\title{
Utilización de supresores de la secreción ácida en pacientes hospitalizados
}

\author{
A. NOGUERADO ASENSIO, R. RODRÍGUEZ BARRIENTOS, P. ZELAYA CASTRO, \\ A. SÁNCHEZ SEMPERE, F. ANTUÑA BLANCO, E. LUTZ GARCÍA, R. QUINTANA \\ DE LA CRUZ, F. ESTELLÉS PIERA, M. S. ALCÁZAR
}

\author{
Servicio de Medicina Interna V. Hospital General Universitario Gregorio Marañón \\ (Cantoblanco). Madrid
}

\author{
USE OF ACID-SUPPRESIVE MEDICATIONS IN HOSPITALIZED \\ PATIENTS
}

\section{RESUMEN}

Introducción: Los fármacos supresores de la acidez gástrica son frecuentemente utilizados, pero algunos estudios evidencian su mal uso, sobretodo en la profilaxis de la úlcera de estrés en pacientes con bajo riesgo. Este estudio evalúa su frecuencia y utilización de en pacientes hospitalizados en una planta de Medicina Interna.

Material y métodos: Revisión aleatoria y retrospectiva de 209 historias clínicas de 661 pacientes ingresados durante el año 2000 en el servicio de Medicina Interna V Hospital General Universitario Gregorio Marañón (Cantoblanco). Definiciones: Utilización: Al menos una vez, a cualquier dosis y de cualquier fármaco antiácido. Indicación correcta: Criterios endoscópicos: Ulcera activa, Esofagitis y Gastritis. Criterios clínicos: síntomas de reflujo, dispepsia, mantenimiento por proceso previo, hemorragia digestiva alta activa, ingesta de antiinflamatorios no esteroideos, profilaxis de úlcera de estrés en pacientes con alto riesgo. Indicación incorrecta: Cualquier otra utilización no incluida anteriormente.

Resultados: De 209 pacientes, 75,1\% (157) los utilizaron, edad media de 76 años (23-99), mujeres $89(56,7 \%)$ y hombres 68 $(43,3 \%)$.Los diagnósticos mas frecuentes al ingreso: ICC $34(21,7 \%)$, Neumonía $26(16,6 \%)$, ITU $24(15,3 \%)$ y EPOC $18(11,5 \%)$. La utilización en planta fue de $84,7 \%$ (133), con uso incorrecto $72,2 \%$ (96), la causa de uso incorrecto "sin razones" en 52,1\%(50) y la medicación inhibidores bomba 46,9\% (45).

Conclusiones: Este estudio evidencia una alta utilización de fármacos inhibidores de la secreción ácida, de manera incorrecta en pacientes hospitalizados.

PALABRAS CLAVE: Inhibidores bomba protones. Antagonistas receptores 2 histamina.

\begin{abstract}
Introduction: The acid-suppressive medications are used frequently in hospitalized patients, but some studies suggests that their are overu sed, especially in the treatment of stress ulcer prophylaxis in low-risk patients. This research determine the frequency of use and indications of acid-suppressive medications in hospitalized patients in a internal medi cal service.

Materials and methods: $A$ at random and retrospective revision of 209 medical records of 661 hospitalized patients during the year 2000 in the V Internal Medical Service of the General University Hospital of Gregorio Marañón (Cantoblanco). Definitions: Use: any prescription of an acid-suppressive medication, regardless of dosage regimen, in which the patient received at least one dose. Appropriated indication: endosco pical criteria: treatment of active ulcer disease, esophagitis or gastritis. Medical criteria: symptomatic gastroesophageal reflux, dyspepsia, pro longing of previous treatment, as a part of a treatment regimen for $\mathrm{H}$. pylori, relief of dyspepsia caused by nonsteroidal anti-inflammatory drugs, stress ulcer prophylaxis in high risk patients. Inappropriate indi cation: any other uses were considered not indicated.

Results: Of the 209 patients, a total of $157(75,1 \%)$ received acidsuppressive therapy, average age 76 years old, 89 women (56,7\%) and 68 men $(43,3 \%)$. The admitting diagnoses for the admission were: $\mathrm{CHF}$ 34 (21,7\%), Pneumonia (16,6\%), UTI (15,3\%) y COPD 18 (11,5\%). The use in hospital ward was $84,7 \%$ (133), with inappropriate use $72,2 \%$ (96), the cause of prescription "no reason" $52,1 \%$ (50), and received proton pump inhibitors 46, 9\% (45).

Conclusions: This study show a high frequency of incorrect use of acid-suppressive medications in hospitalized patients.
\end{abstract}

KEY WORDS: Proton pump inhibitors. Histamine 2 receptor antago nists.

Noguerado Asensio A, Rodríguez Barrientos R, Zelaya Castro P, Sánchez Sempere A, Antuña Blanco F, Lutz García E, Quintana de la Cruz R, Estellés Piera F, Alcázar MS. Utilización de supresores de la secreción ácida en pacientes hospitalizados. An Med Interna (Madrid) 2002; 19: 557-560.

\section{INTRODUCCIÓN}

Los fármacos supresores de la acidez gástrica (FSAG) como antiácidos, antagonistas de los receptores tipo 2 de la histamina (Anti-H2), inhibidores de la bomba de protones y el sucralfato como protector de barrera, son utilizados frecuentemente en el ámbito extrahospitalario (1-4), así como en pacientes ingresados (5).

Existen claras y consensuadas indicaciones para su utilización (6-10) pero algunos estudios evidencian su mal uso,

Trabajo aceptado: 11 de junio de 2002

Correspondencia: Arturo Noguerado Asensio. Servicio de Medicina Interna V. (Cantoblanco) Hospital General Universitario Gregorio Marañón. Carretera de Colmenar Viejo, km 14,600. 28049 Madrid. 
sobre todo en la profilaxis de la ulcera de estrés en pacientes con bajo riesgo (11-18). Su utilización en la profilaxis de sangrado digestivo esta aceptado en pacientes de alto riesgo ingresados en cuidados intensivos con ventilación mecánica o alteraciones severas de la coagulación. (19-21)

El objetivo de este estudio es evaluar la frecuencia y utilización correcta o no de FSAG en pacientes hospitalizados en un servicio de Medicina Interna.

\section{MATERIAL Y MÉTODOS}

Se realiza una revisión aleatoria y retrospectiva de 209 historias clínicas de 661 pacientes ingresados durante el año 2000 en el Servicio de Medicina Interna V, Hospital General Universitario Gregorio Marañón (Cantoblanco). Se revisaron los informes previos existentes, la historia de urgencias y de evolución en planta, las hojas de enfermería de urgencias y planta y el informe final de alta. Se utilizaron las siguientes definiciones: a) Utilización: Al menos una vez, a cualquier dosis y de cualquier fármaco supresor de la acidez gástrica. b)El ácido acetil salicílico (ASS) no se considero como antiinflamatorio a dosis inferior a $325 \mathrm{mg}$. c) Los esteroides fueron considerados si se utilizaban a dosis superiores a $15 \mathrm{mg}$ por vía oral o parenteral. d) Indicación correcta: Se aceptaron los criterios seguidos por Nardino et al. (5). a) criterios endoscópicos: úlcera activa, esofagitis y gastritis; b) criterios clínicos: síntomas de reflujo, dispepsia, mantenimiento por proceso previo, hemorragia digestiva alta activa, ingesta de antiinflamatorios no esteroideos (AINEs), profilaxis de úlcera de estrés en pacientes con alto riesgo (pacientes con ventilación mecánica prolongada o alteraciones severas de la coagulación), y c) indicación incorrecta: cualquier otra utilización no incluida anteriormente.

Para el análisis de los datos se utilizó el programa EPiINFO 2000 versión 1.1.

\section{RESULTADOS}

En la tabla I se describen las características generales de los pacientes. De las 209 historias clínicas revisadas, 157 pacientes $(75,1 \%)$ estuvieron en tratamiento con FSAG. La edad media fue de 76 años (23-99), con 89 mujeres $(56,7 \%)$ y 68 hombres $(43,3 \%)$. Los diagnósticos previos más frecuentes fueron: Insuficiencia cardiaca (ICC) 61 (25,7\%), Enfermedad pulmonar obstructiva crónica (EPOC) $53(22,4 \%)$ y Diabetes mellitus (DM) 46 (19,4\%). Los diagnósticos más frecuentes al ingreso fueron ICC $34(21,7 \%)$, Neumonía 26 (16,6\%), Infección tracto urinario (ITU) 24 (15,3\%) y EPOC 18 (11,5\%).

La utilización de FSAG previamente, en urgencias, planta y al alta se expresan en la tabla II. Es destacable que solo $26,8 \%$ de los pacientes estaban en tratamiento con estos fármacos y que durante su estancia en urgencias se incremento a $47,1 \%$, elevándose a $72,2 \%$ en planta y con un leve descenso $65,9 \%$ a su alta del centro.

Con respecto al uso incorrecto de esta medicación no existieron diferencias significativas tanto antes de llegar al hospital 61,9\% (26), en urgencias del 77\% (57), en planta del 72,2 $\%$ (96) y al alta $54,1 \%(85) .(\mathrm{p}=0,1)$.

La causa más frecuente para la utilización incorrecta del fármaco es de razón desconocida, es decir, no se encontró un
TABLA I

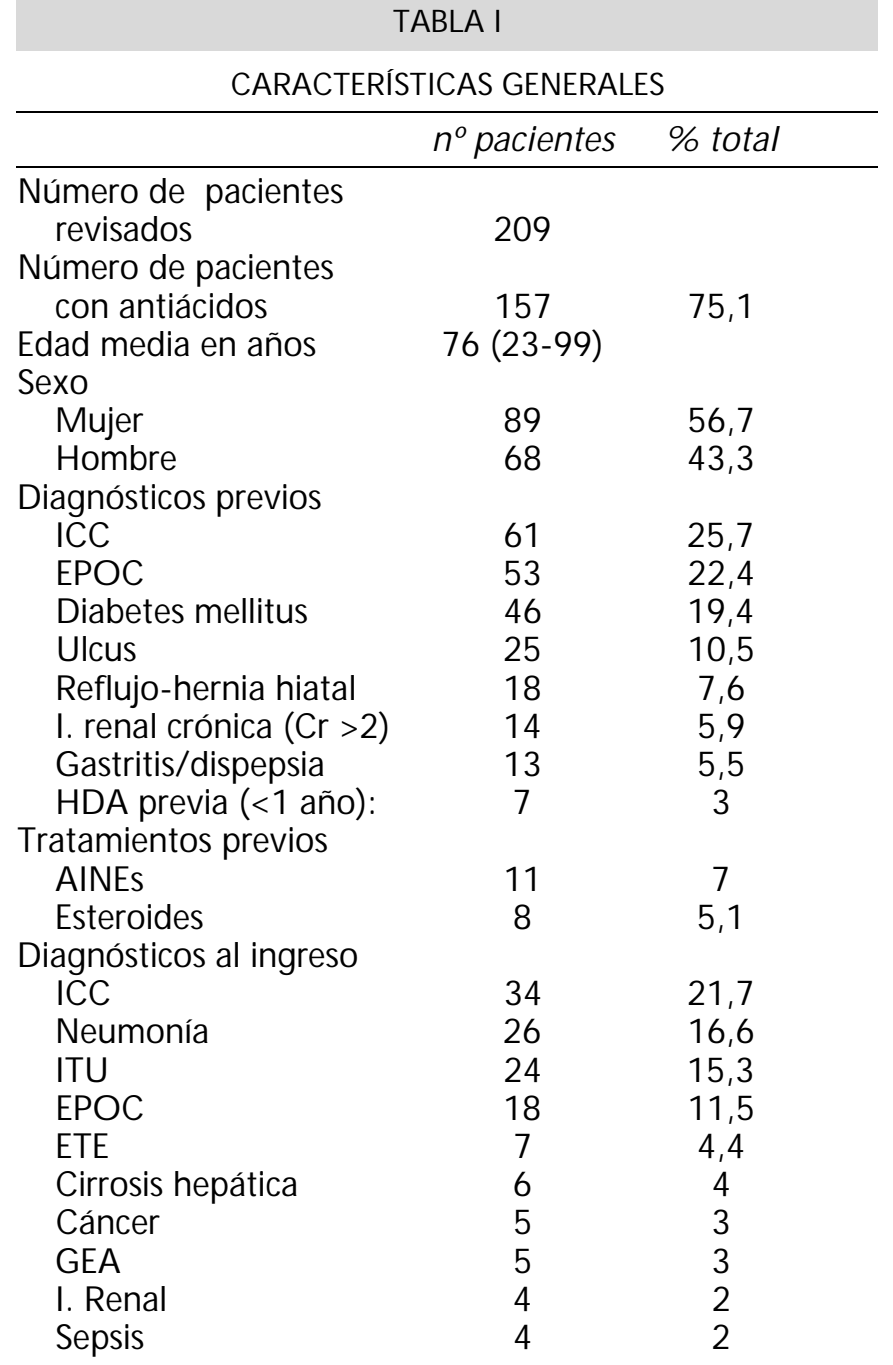

ICC: Insuficiencia cardiaca congestiva. EPOC: Enfermedad obstructiva crónica. HDA: Hemorragia digestiva alta. AINES: Antiinflamatorios no esteroideos. ITU: Infección tracto urinario. ETE: Enfermedad tromboembólica. GEA: Gastroenteritis aguda.

motivo determinado a la hora de prescribir el fármaco. Previamente fue del $57,7 \%$ (15), en urgencias 56,1\% (32), en planta $52,1 \%(50)$ y al alta $44,6 \%$ (25). Sin embargo, al alta la utilización de esteroides fue una causa también importante de utilización incorrecta en el 41,1\% (23).

Los anti-H2 fueron los fármacos más utilizados de manera incorrecta previamente, en urgencias y al alta con un 42,3\% (11), 68,4\% (39) y 55,4 \% (31) respectivamente. En la planta fue más frecuente la utilización incorrecta de los inhibidores de bomba de protones con un 46,9\% (45).

En un análisis mas profundo, es sorprendente observar que de los 26 pacientes que utilizaban previamente y de manera incorrecta estos fármacos, se mantuvo en urgencias a 19, en planta a 15 y al alta seguían 8 , es decir, el 30,7\%. De los pacientes que previamente no tomaban fármacos (115), en urgencias se les instauró en $44(38,3 \%)$,de los cuales en 38 $(86,4 \%)$ fue de manera incorrecta. Así mismo, de los 71 pacientes que no habían recibido medicación previamente ni tampoco en urgencias, se les instauró tratamiento en 67 (94,3\%) 


\begin{tabular}{c} 
TABLA II \\
UTILIZACIÓ N DE ANTIÁCIDOS PREVIAM ENTE, EN \\
URGENCIAS, EN PLANTA Y AL ALTA \\
\hline no pacientes \% total \\
\hline
\end{tabular}

\section{Previa}

Utilización

$\begin{array}{lcc}\text { Sí } & 42 & 26,8 \\ \text { No } & 115 & 73,2 \\ \text { Uso } & & \\ \quad \text { Incorrecto } & 26 & 61,9 \\ \quad \text { Correcto } & 16 & 38,1 \\ \text { Causa uso incorrecto } & & \\ \quad \text { No razones } & 15 & 57,7 \\ \quad \text { AAS antiagregante } & 6 & 23,1 \\ \quad \text { Esteroides } & 4 & 15,4 \\ \quad \text { Profilaxis bajo riesgo } & 1 & 3,8 \\ \text { M edicación uso incorrecto } & & \\ \quad \text { Inhibidores bomba } & 11 & 42,3 \\ \text { AntiH2 } & 11 & 42,3 \\ \text { Antiácidos } & 4 & 15,4\end{array}$

\section{Urgencias}

Utilización

$\begin{array}{lcc}\mathrm{Si} & 74 & 47,1 \\ \text { No } & 83 & 52,9 \\ \text { Uso } & & \\ \quad \text { Incorrecto } & 57 & 77 \\ \quad \text { Correcto } & 17 & 23 \\ \text { Causa uso incorrecto } & & \\ \quad \text { No razones } & 32 & 56,1 \\ \text { Esteroides } & 17 & 29,8 \\ \text { AAS antiagregante } & 5 & 8,8 \\ \text { O tras } & 2 & 3,5 \\ \text { Sangrado previo > 1 año } & 1 & 1,8 \\ \text { M edicación uso incorrecto } & & \\ \text { AntiH2 } & 39 & 68,4 \\ \text { Inhibidores bomba } & 16 & 28,1 \\ \text { Antiácidos } & 2 & 3,5\end{array}$

\section{Planta}

Utilización

\begin{tabular}{lcc} 
Si & 133 & 84,7 \\
No & 24 & 15,3 \\
Uso & & \\
$\quad$ Incorrecto & 96 & 72,2 \\
$\quad$ Correcto & 37 & 27,8 \\
Causa uso incorrecto & & \\
$\quad$ No razones & 50 & 52,1 \\
Esteroides & 31 & 32,3 \\
Otras & 6 & 6,3 \\
AAS antiagregante & 4 & 4,2 \\
Profilaxis de bajo riesgo & 3 & 3,1 \\
Pancreatitis aguda & 1 & 1 \\
Hemorragia digestiva baja & 1 & 1 \\
Medicación uso incorrecto: & & \\
Inhibidores bomba & 45 & 46,9 \\
AntiH2 & 44 & 45,8 \\
Antiácidos & 5 & 5,2 \\
Sulcralfato & 2 & 2,1 \\
\hline
\end{tabular}

\begin{tabular}{|c|c|c|}
\hline \multicolumn{3}{|c|}{ TABLA II (CONT.) } \\
\hline & $\mathrm{n}$ - pacientes & $\%$ total \\
\hline \multicolumn{3}{|l|}{ Alta } \\
\hline \multicolumn{3}{|l|}{ Utilización } \\
\hline $\mathrm{Si}$ & 85 & 54,1 \\
\hline No & 72 & 45,9 \\
\hline \multicolumn{3}{|l|}{ Uso } \\
\hline Incorrecto & 56 & 65,9 \\
\hline Correcto & 29 & 34,1 \\
\hline \multicolumn{3}{|l|}{ Causa uso incorrecto } \\
\hline No razones & 25 & 44,6 \\
\hline Esteroides & 23 & 41,1 \\
\hline AAS antiagregante & 3 & 5,4 \\
\hline Profilaxis de bajo riesgo & 2 & 3,6 \\
\hline Otras & 2 & 3,6 \\
\hline Pancreatitis aguda & 1 & 1,8 \\
\hline \multicolumn{3}{|l|}{ M edicación uso incorrecto } \\
\hline AntiH2 & 31 & 55,4 \\
\hline Inhibidores bomba & 23 & 41,1 \\
\hline Antiácidos & 2 & 3,6 \\
\hline
\end{tabular}

durante su ingreso en la planta siendo en $50(74,6 \%)$ de manera incorrecta, no existiendo diferencias significativas $(\mathrm{p}=0,1)$.

\section{DISCUSIÓN}

Este estudio evidencia una alta utilización de fármacos supresores de la secreción ácida, de manera incorrecta en pacientes hospitalizados.

Nuestros resultados son similares a los publicados en otras series (5,11,13-16). Así, Nardino et al (5) observa que un 54\% de los pacientes hospitalizados estaban en tratamiento con estos fármacos, que el $65 \%$ de las prescripciones fueron incorrectas y que al alta, el $67 \%$ de los pacientes seguían tomando tratamiento de manera innecesaria. En pacientes no ingresados en cuidados intensivos, Carmona-Sanchez et al (13) encuentran que un $76 \%$ de los pacientes recibían anti-H2 de manera profiláctica, de los cuales sólo estaba justificado en un $35 \%$. Gullota et al (11) realiza un estudio multicéntrico transversal con 3685 pacientes ingresados observando que el $26,8 \%$ estaban en tratamiento, de ellos, el $73 \%$ para profilaxis de ulcus péptico y en el 51,4\% de los pacientes su utilización era incorrecta.

Otros estudios realizados con pacientes ingresados en cuidados intensivos evidencian el mismo problema sobre todo en la utilización de profilaxis para la úlcera de estrés $(12,15)$. Así, Lam et al (12) en un cuestionario realizado sobre 328 médicos, concluye que existe escaso consenso en la utilización de estos fármacos, que se utilizan por largos periodos de tiempo sin claras indicaciones y que el costo es excesivo.

Ante esta situación, se han realizado estudios de intervención (22-24), donde se observa que la implantación de guías clínicas se asocia a un incremento en su correcta utilización y disminución de los costes sin aumento de sangrado digestivo.

Sorprende, que en este estudio la causa más frecuente de la 
utilización incorrecta no tenga ninguna razón justificada, aunque muy probablemente pensamos que esté encubierta una profilaxis en pacientes de bajo riesgo como en el estudio de Nardino et al. (5)

La utilización de esteroides para diferentes procesos conlleva la implantación de fármacos supresores de la acidez gástrica como profilaxis de sangrado gastrointestinal. Esta práctica es bastante común pero no hay evidencia científica que sugiera esta asociación (25). En nuestro estudio, supone un tercio en todas las utilizaciones incorrectas en pacientes hospitalizados y un 41,1\% al alta. Según Goldstein (26), sólo en pacientes que toman concomitantemente AINEs estarían indicados como profilaxis.

Los fármacos anti-H2 son los mas frecuentemente utilizados, aunque en nuestra serie, en el paciente ingresado en plan- ta, es algo más frecuente la utilización de inhibidores de la bomba de protones.

En resumen, ante la utilización excesiva de estos fármacos y de manera tan incorrecta, se deberían implantar guías de actuación en cada centro y en diferentes áreas de hospitalización y posteriormente evaluadas, para intentar mejorar el uso de estos fármacos y disminuir el impacto económico de su utilización incorrecta.

\section{AGRADECIMIENTOS}

A los doctores E. Díaz Argüelles, S. Martín Álvarez, T. Chi Anderson y C. Martín Fuertes por su colaboración.

\section{Bibliografía}

1. Thors H, Sigurdsson H, Oddsson E, Thjodleifsson B. Survey of prescriptions for peptic ulcer drugs (ACT class AOB2) in Iceland. Scand J Gastroenterol 1994; 29: 988-94.

2. Zambrana García JL, Martín Escalante MD. Use and abuse of the prescription of anti-ulcer agents. Aten Primaria 2000; 26: 711-2.

3. Roberts SJ, Bateman DN. Prescribing of antacids and ulcer-healing drugs in primary care in the north of England. Aliment Pharmacol Ther 1995; 9: 137-43.

4. Erviti J. Multicenter study of prescription-indication of antacids and antiulcer agents in primary care. Aten Primaria 1999; 24: 134-9.

5. Nardino RJ, Vender RJ, Herbert PN. Overuse of acid-suppressive therapy in hospitalized patients. Am J Gastroenterol 2000; 95: 3118-22.

6. Souney PF. Stress ulcer prophylaxis in an evidence-based analysis of therapy. Am J Gastroenterol 1999; 94: 2566-7.

7. ASHP Therapeutic Guidelines on Stress Ulcer Prophylaxis. ASHP Commission on Therapeutics and approved by the ASHP Board of Directors on November 14, 1998. Am J Health Syst Pharm 1999; 56: 347-79.

8. Tryba M, Cook D. Current guidelines on stress ulcer prophylaxis. Drugs 1997; 54: 581-96.

9. Maton PN, Burton ME. Antacids revisited: a review of their clinical pharmacology and recommended therapeutic use. Drugs 1999; 57: 85570.

10. Estruch R, Pedrol E, Castells A, Masanes F, Marrades RM, UrbanoMarquez A. Prophylaxis of gastrointestinal tract bleeding with magaldrate in patients admitted to a general hospital ward. Scand J Gastroenterol 1991; 26: 819-26.

11. Gullotta R, Ferraris L, Cortelezzi C, Minoli G, Prada A, Comin U et al.Are we correctly using the inhibitors of gastric acid secretion and cytoprotective drugs? Results of a multicentre study. Ital J Gastroenterol Hepatol 1997; 29: 325-9.

12. Lam NP, Le PD, Crawford SY, Patel S. National survey of stress ulcer prophylaxis. Crit Care Med 1999; 27: 98-103.

13. Carmona-Sánchez R, Suazo-Barahona J, González A, Carmona-Sánchez L, Uscanga-Domínguez L. Use and abuse histamine $\mathrm{H} 2$ receptor blockers in hospitalized patients. Rev Gastroenterol Mex 1997; 62: 84-8.

14. Erstad BL, Barletta JF, Jacobi J, Killian AD, Kramer KM, Martin SJ.

Survey of stress ulcer prophylaxis. Crit Care (Lond) 1999; 3(6): 145149.

15. Pillans PI, Kubler PA, Radford JM, Overland V. Concordance between use of proton pump inhibitors and prescribing guidelines. Med J Aust 2000; 172: 16-8

16. Dettmer RM, Riley TH, Byfield F, Green PH. The use of intravenous H2-receptor antagonists in a tertiary care hospital. Am J Gastroenterol 1999; 94: 3473-7.

17. Naunton M, Peterson GM, Bleasel MD. Overuse of proton pump inhibitors. J Clin Pharm Ther 2000; 25: 333-40.

18. Ryder SD, O'Reilly S, Miller RJ, Ross J, Jacyna MR, Levi AJ. Long term acid suppressing treatment in general practice. BMJ 1994; 308: 827-30.

19. Cook DJ. Stress ulcer prophylaxis: gastrointestinal bleeding and nosocomial pneumonia. Best evidence synthesis. Scand J Gastroenterol Suppl 1995; 210: 48-52.

20. Kress S, Schilling D, Riemann JF. Concept of stress ulcer prevention. Is re-thinking necessary? Med Klin 1998; 93: 486-91.

21. Cook DJ, Reeve BK, Guyatt GH, Heyland DK, Griffith LE, Buckingham L et al. Stress ulcer prophylaxis in critically ill patients. Resolving discordant meta-analyses. JAMA 1996; 275: 308-14.

22. Erstad BL, Camamo JM, Miller MJ, Webber AM, Fortune J. Impacting cost and appropriateness of stress ulcer prophylaxis at a university medical center. Crit Care Med 1997; 25: 1678-84.

23. Pitimana-aree S, Forrest D, Brown G, Anis A, Wang XH, Dodek P. Implementation of a clinical practice guideline for stress ulcer prophylaxis increases appropriateness and decreases cost of care. Intensive Care Med 1998; 24: 217-23.

24. Devlin JW, Ben-Menachem T, Ulep SK, Peters MJ, Fogel RP, Zarowitz BJ. Stress ulcer prophylaxis in medical ICU patients: annual utilization in relation to the incidence of endoscopically proven stress ulceration. Ann Pharmacother 1998; 32: 869-74.

25. Conn HO, Poynard T. Corticosteroids and peptic ulcer: meta-analysis of adverse events during steroid therapy. J Intern Med 1994; 236: 619-32.

26. Goldstein JL. Who needs prophylaxis of nonsteroidal anti-inflammatory drug-induced ulcers and what is optimal prophylaxis? Eur J Gastroenterol Hepatol 2000; 12 Suppl 1: S11-5. 